\title{
An Analytical Framework of Division, Design \& Development of Consciousness
}

\author{
Gopal Krishna Sharma ${ }^{1}$, Dr. Pranav Pandya ${ }^{2}$, and Dr. Abhay Saxena ${ }^{3}$
}

\begin{abstract}
Consciousness has always been treated as a clandestine since epochs. Its studies scaled broader in different disciplines and approaches makes the same more convoluted. Considering consciousness more experiential and subjective researches are done but has always ended with new questions. The material sciences have done significant progress in the time being. Human behavior has an intricate relation with brain and its functions. Biologists, Neuroscientists have tried to explain brain its anatomy and physiology in order to understand human behavior. Psychology as a science of studying human behavior attempts to answer few basic questions relates the human black box (brain) but many are still unanswered. The physical artifacts and devices too analogously mimic humans as it was done in the case of Central Processing Unit (CPU) in the computer. Computers Scientists are making serious attempts to formulate Artificial Consciousness. Present paper attempts to provide a comprehensive understanding of division, design and development of Consciousness. The paper also provides a better understanding regarding the paradigm shift of human consciousness studies towards Machine consciousness simulation. Lastly with the help of available models of consciousness, we are trying to evolve another consciousness model.
\end{abstract}

Keywords-Consciousness, Machine Consciousness, Artificial Consciousness, Human Machine Interaction.

\section{INTRODUCTION}

$\mathrm{C}_{\mathrm{s}}^{\mathrm{os}}$ ONSCIOUSNESS is always considered as an arcane mystery still to be resolved. With the development of science and technology researchers from different disciplines other than philosophy, psychology took interest and started exploring the underlying enigma of consciousness. Consciousness basically attributed to Humans and their behaviour. It is considered to have an intricate relation with the neural structure of the brain and its functions. Biologists, Neuroscientists have tried to explain brain, its anatomy and physiology in order to understand human behaviour. What seems visible, logically identifiable

${ }^{1}$ Research Scholar, Department of Computer Science, Dev Sanskriti Vishwavidyalya, Uttarakhand, India

${ }^{2}$ Honorable Chancellor, Dev Sanskriti Vishwavidyalya, Uttarakhand, India

${ }^{3}$ Head, Department of Computer Science, Dev Sanskriti Vishwavidyalya, Uttarakhand, India. and deductible to them they explored but certain phenomenon far away from the realms of basic understanding incurring complex functions and structured are still under study. Consciousness is one such major phenomena which could be felt and defined but is more subjective in nature and lacks objectivity and generality. A prominent hallmark of consciousness is individual's awareness of his thought, which widely varies among individuals. Intelligence is one such phenomena of the human brain which has been explored in a way to get and objective representation away from the barriers of subjectivity. Intelligence in human perspective found a new way as Artificial Intelligence in computer's or machine's perspective. Artificial Intelligence is the intelligence exhibited by machines or software.

AI is an area of research which studies how to create computers and computer software that care capable of intelligent behaviour. Intelligence is an area of study for psychology, cognitive sciences and neuroscience Artificial Intelligence incorporates the previous ones and of computer science as well which attempts at "the study and design of intelligent agents." Question of division, design and development of an analytical framework of consciousness relates to division, design and development of an analytical framework of Intelligence. Intelligence in its natural existence in humans is a field of study of its own but it also helps to mimic it for artificial systems- computer, machines. It's not about recreation of Intelligence rather drawing analogies and inferences in order to program computers based on algorithms and languages. Consciousness as a natural phenomenon is unique but with varied definitions. One agreed definition of consciousness relates it with awareness. Consciousness in order to divide, design and develop as an analytical framework needs to be understood in segments and as it was done with Intelligence the same way attempt could be made in the direction of mimicking the features of Consciousness in machines as Artificial Consciousness.

\section{COMPUTER, INTELLIGENCE AND CONSCIOUSNESS - QUiCK HISTORICAL REVIEW}

To analyse the problem of present research, need is to look long back in the history of mankind. Human capacity and capability of the brain is tremendous but in order to get assistance scientists decided to develop a machine in order to facilitate ease in calculation. Calculation is a task performed directly by human brain through its inhibiting ability of intelligence. Psychology studies intelligence in functional way 
into deeper realms but its aspect of calculation, logical deduction and comparison finds its proximity with mathematics. The earliest known tool for use in computation was the abacus, developed in period 2700-2300 BC in Sumer. Mathematical modelling of the identified theories and constructs paved a way towards development of the machine popularly known as Computer, which started calculations of complex series of problems and brought an ease to human endeavour. Mechanical Analog Computers came further as the developed mathematical model paved a new way further. According to the Barnhart Concise Dictionary of Etymology (Robert Barnhart, ed., NY: Harper Collins, 1995), computer came into use in English in 1649 as a word for a 'one who computes' and then by 1897 as a 'mechanical calculating machine'.

In early 1950's Alan Turing a famous mathematician, logician, cryptanalyst who played highly influential role in the development of computer science, providing formalisation of the concepts of algorithm and computation with the Turing Machine, which is considered as a model of a general purpose computer. He is widely considered to be the father of theoretical computer science and artificial intelligence talked about the idea, "Can machine think?" in his research work "Computing machinery and intelligence" (Turing, A.M. (1950). Consider 1950's when Turing actually talked about the idea and its 2015 when actualization of his idea which was criticised by various schools of thoughts that time is in real. In the evolution of computer as a machine, computer as a science and computer science as a discipline of study it has undergone various phases in generations.

The way Charles Babbage "father of the computer" looked at the concept of computing was of a machine which not only inputting or outputting rather processing and storing too. Konrad Zuse is considered to be the creator of first electromechanical binary programmable (modern) computer and really the first functional computer. Von Neumann Architecture described design architecture for an electronic digital computer with parts consisting of a various processing units, mass storage, input and output. Gordon E. Moore, the co-founder of Intel gave Moore's law which says that the number of transistors in a dense integrated circuit doubles approximately every two years.

Yet on 50 Years of Moore's Law, Gordan said, "We won't have the rate of progress that we've had over the last few decades. I think that's inevitable with any technology; it eventually saturates out. I guess I see Moore's law dying here in the next decade or so, but that's not surprising (Moore, 2015)." Central Processing Unit (CPU) is called brain of the computer and is given equal importance that of brain in humans. Intelligence rather was not taken completely rather as a function it was opted. Artificial Intelligence through its approaches, methods, tools is progressive but few strong AI problems opened new realms of thinking.

\section{TOWARDS ARTIFICIAL CONSCIOUSNESS}

Computer developed with interdisciplinary contribution and development of science. It developed as a system with distinct faculties but integrated solution. Analogous to the human brain in early 1950's Jon McCarthy the computer scientist coined the term Artificial Intelligence in 1955 defined it as "the science and engineering of making intelligent machines". Computer which actually evolved for computing further expanded its areas into mimicking human intelligence. Intelligence in parts can be logical and algorithmic as well. Artificial Intelligence attempts at programming deduction, reasoning, problem solving, knowledge representation, learning and natural language processing and perception etc. in the best possible manner in order to get optimum results.

Intelligence cannot be limited to structure and function. Awareness of the real time plays a vital role in Intelligence too. Scientists started working in the area of intelligence agents. Agents for better functioning required effective interaction with the environment. Awareness is a functional major of consciousness in human's perspective and can be simulated in the same way for computer's perspective. Artificial Consciousness is a stead ahead but in the realms of Artificial Intelligence. Pentti O. Haikonen (2003), talked that "One of these days, not very far away in the future a machine may, after a thorough reflection, reach the conclusion : "I think; therefore I am.....immaterial". In order to proceed ahead in Artificial Intelligence research Artificial Consciousness plays a pivot role.

(Sanz 2005) had given three motivations to pursue AI. a) implementing and designing machines resembling human beings (cognitive robotics) b) Understanding the nature of consciousness (cognitive science) and c) Implementing and designing more efficient control systems

Artificial Consciousness, sometimes labeled as machine consciousness, is the attempt to model and implement those aspects of human cognition which are identified with the often elusive and controversial phenomenon of consciousness (Aleksander 2008, Chella and Manzotti 2009). The aim of the theory of artificial consciousness is to, "Define that which would have to be synthesized were consciousness to be found in an engineering artifact." (Aleksander 1995). Artificial Consciousness is not about recreating Human Consciousness.

IBM developed computer 'Watson' won the TV game show, Jeopardy, in 2011 by answering general knowledge questions posted in natural language. It has more recently been used as a customer call centre agent. Apple SIRI, Google Now, Google Photo Search and Google's Self-Driving Cars exhibits behavior similar to that exhibited by intelligent humans. Google is in the best position to create the first conscious machine. Consciousness is a step beyond machine intelligence seen in IBM's Watson supercomputer. A conscious machine aware about its existence, environment and self could identify and correct errors without being asked about it.

\section{A. Studies in Artificial Consciousness}

Artificial Consciousness became an area of study for investigators of different disciplines. In order to have better idea of the basic theoretical concept to model consciousness 
for machines correlates are explored and even to assess and measure it approaches are in study. Consciousness

\section{B. Studies in Artificial Consciousness unfold the following subareas:}

\section{- Theoretical Foundation of Artificial Consciousness}

Area which studies about the underlying theoretical concepts which will pave our way towards better understanding of consciousness. We are not attempting at designing or developing overall perspective of consciousness at once rather attributing to specifically identified functionalities. It incorporates Defining Consciousness, drawing inferences through understanding definitions of different disciplines, types of consciousness, types identified in the context of computational modelling in use - A-Consciousness, PConsciousness etc., philosophical perspectives and various other theories related with consciousness.

\section{- Computational Model of Artificial Consciousness}

Modelling consciousness demands better understanding of the various segments and functionalities of consciousness which we can bring into action through our model. Consciousness with its diversified domain of study explores models based on certain proposed theory, certain functions, few individual components, complex processes etc. Cognition has got major attentions in many models. Computational modelling is dominated by cognitive aspects of consciousness.

\section{- Correlates of Artificial Consciousness}

Computer is a system in certain conditions replica of human phenomenon of consciousness. It is also known as awareness which is the by product of interaction with several sub systems. Whether consciousness can be created with silicon chips or it is based only on biological brain and neurons is a big question and to get answer to these questions studies are done to identify correlates of Artificial Consciousness. Researches talks about Biological Correlates of Consciousness (BCC), Neural Correlates of Consciousness (NCC), Computational Correlates of Consciousness (CCC) etc.

\section{- Measurement and Assessment of Artificial Consciousness}

Consciousness as an attribute can be logically presented into its different subsections. Measurement and assessment of which is done through certain means. It actually verifies the applicability of the model. It has two distinct dimensions one about measuring human consciousness in order to understand underlying concept and other is measuring machine or artificial consciousness to verify its functioning and applicability in machines. ConScale (Raul A., 2010) is one such example which is used to measure machine consciousness.

\section{- Possibilities and ways to achieve Conscious Machine}

Progress in Artificial Consciousness has raised the hopes of materializing the dreams into reality. Based on the above areas of studies on certain theories, definitions, functions, models, frameworks, correlates, measurement, assessment possible ways are opted. Tried and error method gives learning. With advent of hardware, software and programmes ways are tested on machine and further improved in order to attain Conscious Machine.

\section{ANALytical Framework of ARTIFicial CONSCIOUSNESS}

Research on algorithms and representations once dominated AI. Recently the importance of architecture has been acknowledged, but researchers have different objectives, presuppositions and conceptual frameworks, and this can lead to confused terminology, argumentation at cross purposes, reinvention of wheels and fragmentation of the researcher (Sloman, 2008) To understand the concept beneath designing analytical framework of artificial consciousness we need to review the previous contributions. Existing research work done in this direction poses some questions:

- Investigators use terms consciousness and artificial consciousness in what sense they use it? In different paper, thesis and articles they are used with bit similar and distinct way.

- We are modeling consciousness or artificial consciousness? As interchangeable use of words in research writings make the things little vague.

- What are the delimiting criteria to keep in mind while considering certain model or framework

Questions are not only limited to this but in order to proceed ahead in a direction these are few basics to be answered.

\section{A. Computational Models/Architecture/ Framework of Consciousness}

\section{- CogAff Schema [Sloman \& Chrisley, 2003]}

"A methodological framework: develop a general representation of a wide class of architectures within which different architectures can be compared and contrasted. As a first-draft example they presents the CogAff architecture schema, and show how it provides a useful framework for comparing and contrasting a wide range of architectures, including $\mathrm{H}$-Cogaff, a proposed architecture for human-like systems.

\section{- Haikonen's Model of Consciousness [Haikonen, 2003]}

"An attempt to explicate a platform for modelling consciousness. It was developed in three parts: part 1 offers a brief analysis of the state of play in cognitive modelling; part 2 an extended treatment of the phenomena to be explained; part 3 promises a synthesis of the two preceding discussions provide the necessary background and detail for the proposed modelling environment. Final part covers a broad range of technical detail from the nature of the representationalcomputational economy instantiated, to control of motor output, to the means of implementing emotions in artefacts.

\section{- Axiomatic Theory Model of Consciousness [Aleksandar, 2007 ]}


"A model of conscious mechanism called Axiomatic Consciousness Theory $[\mathrm{ACT}]$ is used to develop a theoretical computational model of visual phenomenology. The result is an extension of concepts in AI towards phenomenon intentionality: the lack of which is a common starting point for critiques of AI. Here the argument is developed at four interacting grain levels of computational description and the associated theoretical analysis. The visual domain is highlighted due to its dominance in discussions involving inner mental states".

\section{- CODAM Model [Taylor, 2007 ]}

Attention has been recognized through many experiments to act as a filter processing system. In order to clarify how this might be achieved in general they considered the two sorts of attention that are now appreciated as different in character: top-down (endogenous) and bottom-up (exogenous). CODAM model gives a detailed model of attention control, containing more complex and efficient mechanisms. CODAM thereby has the ability to explain a considerable amount of brainbased data on attention (Korsten et al., 2006). The model also has the ability to enable activity of an ownership character to be located in the model, approach with little modification is called 'attention copy models of consciousness.'(Taylor, 2007)

\section{- LIDA Model [Baar \& Franklin, 2009]}

"LIDA (Learning IDA) model of consciousness and cognition implements Global Workspace Theory (GWT) (Baars 1988, 1997), which has become the most widely accepted psychological and neurobiological theory of consciousness (Baar 20002; Dehaene \& Naccache 2001; Kanwisher 2001). It is a working model of, and theoretical foundation for, machine consciousness. LIDA's architecture and mechanisms were inspired by a variety of computational paradigms. The LIDA architecture's cognitive modules include perceptual associative memory, episodic memory, functional consciousness, procedural memory and actionselection. Cognitive robots and software agents controlled by the LIDA architecture will be capable of multiple learning mechanisms."

\section{- Computational Model of Conscious Machine [Starzyk and Prasad, 2010]}

"The available definitions of consciousness being human centred, vague and incomplete which leads to the notion despite of many efforts, there are no computational models of consciousness that can be used to design conscious intelligent machines Through a biological analysis of consciousness and concept of machine intelligence, they proposed a physical definition of consciousness with the hope to model it in intelligent machines. Further they proposed a computational model of consciousness driven by competing motivation, goals, and attention switching.

\section{- Proposed hybrid model of Consciousness:}

Models of consciousness explains the idea in segments and it is obvious as further integration of all these will collectively give an incremental solution to the development of the conscious machine. The approaches opted while devising different models shows us a path leading towards the way we wanted to proceed. We need to understand the basic definition, then to defragment it into operational terms, and further it needs to identify the correlates their incorporation so that further design and development can takes place. The proposed model will be extension of Computational Model of Conscious Machine [Starzyk and Prasad, 2010] with a blending of the LIDA architecture's cognitive modules include perceptual associative memory, episodic memory in addition with the [Haikonen, 2003] feature of cognitive modelling.

\section{V.CONCLUSION}

Present research concludes on the idea of formation of the proposed model of consciousness based on the finding commonalities of the models, theories present herewith and moving in the direction of cognitive modelling which has got success in previous works. Moore's law prediction about the processing ability of the computer forecasts in order to achieve processing ability similar to neural correlates of our brain that by 2029 it will be possible. Using Artificial Neural Network the connections of neuron and synapses similar to that of human brain which if is considered as Neural Correlates of Consciousness (NCC) could be considered can then be designed in artificial brain silicon chip too. Quantum Computing with its qbits can possess that high end processing that with the help of that hardware, software, intelligent agent, program will function that way that we can achieve artificial consciousness through conscious machines. Predictions are done by Giorgio Buttazzo, Ray Kurzweil, Gregory S. Paul and Earl Cox and Hans Moravec. This idea has been further explored and presented in a paper "Quantum Artificial Consciousness" by (Saxena A., Sharma G.K., 2011). Consciousness to be modelled for computers has to deal with human consciousness with an approach of understanding it and separately choosing the attributes which can be mimicked through present technology it does not mean to compete recreation of consciousness.

\section{REFERENCES}

[1] A. Chella and R. Manzotti. (2007). Artificial Consciousness. Exeter (UK). Imprint Academic.

[2] A. Cleeremans. (2004). Computational correlates of consciousness. In press: Progress in Brain Research, pp.2-42.

[3] A. Saxena and G.K. Sharma. (2013). Quantum Artificial Consciousness. Dev Sanskriti Interdisciplinary International Journal. 2 (1). pp. 13-20.

[4] A. Sloman and R. Chrisley. (2003). Virtual machines and consciousness. [Article]. Journal of Conscious Studies. 10 (4-5). pp.133-172.

[5] A.L. Nelson. (2013). Artificial life and machine consciousness. Available: http://www.aaai.org.

[6] B.J. Baars and S. Franklin. (2009). Consciousness is computational: The LIDA model of global workspace theory. International Journal of Machine Consciousness. 1(1).pp.23-32. http://dx.doi.org/10.1142/S1793843009000050

[7] D. Dermott. (2007). "Artificial Intelligence and Consciousness." Yale University, The Cambridge Handbook of Consciouness. Cambridge University Press, pp.117-150.

[8] D. Gamez. (2008). Progress in machine consciousness: Consciousness and cognition. 17(3). pp.887-910.

http://dx.doi.org/10.1016/j.concog.2007.04.005 
[9] G. Buttazzo. (2001). “Artificial Consciousness: Utopia or Real Possibility.” In Spectrum IEEE Computer. 43 (7). pp. 24-30.

http://dx.doi.org/10.1109/2.933500

[10] G.K. Sharma and A. Saxena, "A study of consciousness from the epochs of humans towards computers," presented at the India-Latvia Conference, June, 2014.

[11] I. Aleksander and H. Morton. (2007). Axiomatic consciousness theory for visual phenomenology in Artificial intelligence. Available: http://www.aaai.org.

[12] I. Aleksander and U. Awret. (2008). Assessing artificial consciousness: A collective review article. Journal of Consciousness Studies. 15 (7). pp. 95110.

[13] J.A. Reggia. (2013). The rise of machine consciousness: Studying consciousness with computational models. Neural Networks. 44. pp. 112131. http://dx.doi.org/10.1016/j.neunet.2013.03.011

[14] J.A. Starzyk and D.k. Prasad. (2010). A computational model of machine consciousness. International Journal of Machine Consciousness. [Online]. Available: http://www.ohio.edu.

[15] J.G. Taylor. (2007). CODAM: A neural network model of consciousness. Neural Networks. 20 (9). pp. 983-992. http://dx.doi.org/10.1016/j.neunet.2007.09.005

[16] P.O.A. Haikonen. (2003). The cognitive approach to conscious machine. UK: Imprint Academic

[17] R .Clowes and S.Torrance. (2007). Machine consciousness-Embodiment and imagination. [Editorial Material]. Journal of Consciousness studies. 14(7). pp.7-14.

[18] R. Arrabales and A. Ledezma. (2008). Criteria for consciousness in Artificial Intelligence agents. AAMAS, Estoril, Portugal.

[19] R. Manzotti. (2012). The computational stance is unfit for consciousness. International Journal of Machine Consciousness. 4 (2). pp. 401-420. Available: http://www.worldscientific.com. http://dx.doi.org/10.1142/S1793843012400239 